\title{
Antimicrobial Resistance - Causes, Implications and Strategies
}

\section{Ravishankar *}

\section{INTRODUCTION}

Antimicrobial agents represent one of the main therapeutic tools both in human and veterinary medicine to control and treat a variety of bacterial infectious diseases. Antimicrobial resistance (AMR) is resistance of a microorganism to an antimicrobial medicine to which it waspreviously sensitive. During the past five decades, the use and sometimes misuse of antimicrobials in both human and veterinary medicine has resulted in the emergence of strains of organisms,which includes bacteria, viruses and some parasitesthat no longer respond to antimicrobial therapy. Not only do antimicrobialresistant bacterial pathogens in animals pose a risk in terms of animal health, they also affect public health when transmitted to humans as foodborne contaminants. Thus, addressing the issue of antimicrobial resistance is one of the most urgent priorities in the fields of public health today.

There are several mechanisms by which bacteria develop resistance to antimicrobial agents and then transfer this resistance to susceptible bacterial strains.

\section{NATURAL MECHANISMS Of ANTIMICROBIAL RESISTANCE DARWINIAN PRINCIPLE}

The increased prevalence and dissemination of resistance is an outcome of natural selection and should be viewed as an expected phenomenon of the Darwinian biological principle of "survival of the fittest."

In any large population of bacteria, a few cells will be present which possess traits that enable them to survive in the presence of a noxious substance, in this case the ability to fend off the action of the antimicrobial. Susceptible organisms, those lacking the advantageous trait, will be eliminated, leaving the remaining resistant populations behind.With longterm antimicrobial use in a given environment / community, the bacterial strains will change dramatically, with more resistant organisms increasing in proportion.

Susceptible bacteria can acquire resistance to antimicrobials by either genetic mutation or by accepting antimicrobial resistant genes from other bacteria.This usually occurs through one of several biochemical mechanisms or by genetic transfer.

The biochemical mechanisms include Mutation, Destruction or Inactivation and Efflux.

\section{MUTATION}

Mutation is a change in the DNA that can sometimes cause a change in the gene product, which is the target of the antimicrobial.

\section{DESTRUCTION OR INACTIVATION}

Many bacteria possess genes which produce enzymes that chemically degrade or deactivate the antimicrobial, rendering them ineffective against the bacterium. Here the antimicrobial is either degraded or modified by enzymatic activity before it can reach the target site and damage the bacterial cell.

\section{EFFLUX}

Certain bacteria can often become resistant to antimicrobials through a mechanism known as Efflux. An efflux pump is essentially a channel that actively exports antimicrobial and other compounds out of the cell.The antimicrobial enters the bacterium through a channel termed a porin, and then is pumped back out of the bacterium by the efflux pump. By actively pumping out antimicrobials, the efflux pumps prevent the intracellular accumulation necessary to exert their lethal activity inside the cell.

The Genetic Transfer is the other mechanism of developing resistance. Genetic material can be transferred between bacteria by several means, most often by Conjugation, Transformation andTransduction.

\footnotetext{
* Dr. M. Ravishankar, Professor and Head,

Anaesthesiology and Critical Care,

MGMC\&RI,

Puducherry
} 


\section{CONJUGATION}

Conjugation is mediated by a particular kind of circular DNA called a plasmid, which replicates independently of the chromosome.Many plasmids carry genes that confer resistance to antimicrobials. When two cells are in close proximity to each other, a hollow bridge-like structure, known as a pilus, forms between two cells. This allows a copy of the plasmid, as it is duplicated, to be transferred from one bacterium to another.This enables a susceptible bacteria to acquire resistance to a particular antimicrobial agent.

\section{TRANSFORMATION}

During this process, genes are transferred from one bacterium to another as "naked" DNA.When cells die and break apart, DNA can be released into the surrounding environment. Other bacteria in close proximity can scavenge this free-floating DNA, and incorporate it into their own DNA. This DNA may contain advantageous genes, such as antimicrobial resistant genes and benefit the recipient cell.

\section{TRANSDUCTION}

In this process, bacterial DNA is transferred from one bacterium to another inside a virus that infects bacteria. These viruses are called bacteriophages or phage.When a phage infects a bacterium, it essentially takes over the bacteria's genetic processes to produce more phage. During this process, bacterial DNA may inadvertently be incorporated into the new phage DNA. Upon bacterial death and lysis (or breaking apart), these new phage go on to infect other bacteria. This brings along genes from the previously infected bacterium.

\section{UNNATURAL / MAN MADE MECHANISMS}

Inappropriate and irrational use of medicines provides favourable conditions for resistant microorganisms to emerge and spread.For example, when patients do not take the full course of aprescribed antimicrobial or when poor quality antimicrobialsare used, resistant microorganisms can emerge and spread.

\section{IMPLICTIONS}

About 440000 new cases of multidrug-resistant tuberculosis (MDR-TB) emerge annually, causing at least 150000 deaths. Extensively drug-resistant tuberculosis (XDR-TB) has been reported in 64 countries to date.

Resistance to earlier generation antimalarial medicines such as chloroquine and sulfadoxine-pyrimethamine is widespread in most malaria-endemic countries. Falciparum malaria parasites resistant to artemisinins are emerging in South-East Asia; infections show delayed clearance after the start of treatment (indicating resistance).

A high percentage of hospital-acquired infections are caused by highly resistant bacteria such as methicillin-resistant Staphylococcus aureus (MRSA) and vancomycin-resistant enterococci.

Resistance is an emerging concern for treatment of HIV infection, following the rapid expansion in access to antiretroviral medicines in recent years; national surveys are underway to detect and monitor resistance.

Ciprofloxacin is the only antibiotic currently recommended by WHO for the management of bloody diarrhoea due to Shigella organisms, now that widespread resistance has developed to other previously effective antibiotics. But rapidly increasing prevalence of resistance to ciprofloxacin is reducing the options for safe and efficacious treatment of shigellosis, particularly for children. New antibiotics suitable for oral use are badly needed.

AMR has become a serious problem for treatment of gonorrhoea (caused by Neisseria gonorrhoeae), involving even "last-line" oral cephalosporins, and is increasing in prevalence worldwide. Untreatable gonococcal infections would result in increased rates of illness and death, thus reversing the gains made in the control of this sexually transmitted infection.

New resistance mechanisms, such as the beta-lactamase NDM-1, have emerged among several gram-negative bacilli. This can render powerful antibiotics, which are often the last defense against multi-resistant strains of bacteria, ineffective.

\section{UNDERLYING FACTORS THAT DRIVE AMR INCLUDE:}

\section{Inadequate national commitment to a}

囚 comprehensive and coordinatedresponse, ill-defined accountability and insufficientengagement of communities.

Weak or absent surveillance and monitoring systems.

Inadequate systems to ensure quality and uninterrupted supplyof medicines.

$\otimes$ Inappropriate and irrational use of medicines, including inanimal husbandry. 
$\otimes$ Poor infection prevention and control practices.

\ Depleted arsenals of diagnostics, medicines and vaccines as well as insufficient research and development on new products.

The emergence of AMR is a complex problem driven by many interconnected factors; single, isolated interventions have little impact. A global and national multi-sectoral response is urgently needed to combat the growing threat of AMR.

\section{Chalenges To Overcome}

$>$ Limited public awareness and government commitment: media attention focuses on individual outbreaks but gives little attention to the wider threat of AMR. It is therefore not a priority of national governments or linked to the achievement of the health related Millennium Development Goals. Despite their potential to influence policy too few nongovernmental, donor and health advocacy agencies have recognized the threat of AMR.

$>$ Fragmentation of effort: specific activities to combat AMR are taken forward by individual programs, institutions and regulatory bodies, but too often in the absence of an overall strategy; without an adequate budget; and with no accountability for results.

$>$ Perverse incentives contribute to AMR, through the unregulated marketing of antimicrobials, their use for growth promotion in livestock, or profit-seeking prescribing of antibiotics in human health care. Where legal frameworks exist they are rarely accompanied by effective sanctions.

$>$ The voiceless are the worst affected: poor people are among those who are at greatest risk of infections and those most likely to lack access to quality services and suffer severe illness. They usually lack information or opportunity to demand or become involved in actions to combat AMR.

\section{Strategies To Combat AmR}

1. Commit to a Comprehensive, financed National plan with accountability and Civil Society engagement.

2. Strengthen surveillance And laboratory capacity

3. Ensure uninterrupted access to essential Medicines of assured quality.

4. Regulate and promote rational use of Medicines, including in animal husbandry, And ensure proper patient care.

5. Reduce use of antimicrobials in food-producing animals

6. Enhance infection prevention and control

7. Foster innovations and research and development for new tools

\section{Why Is A Comprehensive National Plan Essential For Combating Antimicrobial Resistance?}

The factors which favour the emergence and spread of resistant microbes, and the measures needed to combat antimicrobial resistance (AMR), are well known. A comprehensive plan brings all the elements together so that everyone can do more to make a difference:

\section{Policy-makers and planners in government}

$>$ must provide the legal framework and strategy that people need to work together effectively to combat AMR and its public health consequences.

$>$ Civil society and patients' groups can raise awareness of the issues, and demand action by policy-makers and all stakeholders. Everyone can play a part by using prescribed medicines correctly.

$>$ Practitioners and prescribers need to combine appropriate prescription of antimicrobials with clear, accurate and understandable advice to patients. Veterinarians should resist pressures to use antibiotics as growth promoters and for disease prevention in livestock.

$>$ Pharmacists and dispensers must ensure that antimicrobials are safe: obtained from an approved source and stored properly, and supplied in line with prescriptions.

$>$ Providers, institution managers, and communities should apply infection control measures to prevent the emergence and spread of AMR.

$>$ The diagnostic and pharmaceutical industry, through research and development, will need to produce the tools needed to better prevent and detect disease, identify AMR earlier, and develop new antimicrobials needed to replace those that have become ineffective. 